\title{
Research
}

Narinder Chana, Talya Porat, Cate Whittlesea and Brendan Delaney

\section{Improving specialist drug prescribing in primary care using task and error analysis:}

\author{
an observational study
}

\begin{abstract}
\section{Background}

Electronic prescribing has benefited from computerised clinical decision support systems (CDSSs); however, no published studies have evaluated the potential for a CDSS to support GPs in prescribing specialist drugs.

\section{Aim}

To identify potential weaknesses and errors in the existing process of prescribing specialist drugs that could be addressed in the development of a CDSS.
\end{abstract}

\section{Design and setting}

Semi-structured interviews with key informants followed by an observational study involving GPs in the UK

\section{Method}

Twelve key informants were interviewed to investigate the use of CDSSs in the UK. Nine GPs were observed while performing case scenarios depicting requests from hospitals or patients to prescribe a specialist drug. Activity diagrams, hierarchical task analysis, and systematic human error reduction and prediction approach analyses were performed.

\section{Results}

The current process of prescribing specialist drugs by GPs is prone to error. Errors of omission due to lack of information were the most common errors, which could potentially result in a GP prescribing a specialist drug that should only be prescribed in hospitals, or prescribing a specialist drug without reference to a shared care protocol. Half of all possible errors in the prescribing process had a high probability of occurrence.

\section{Conclusion}

A CDSS supporting GPs during the process of prescribing specialist drugs is needed. This could, first, support the decision making of whether or not to undertake prescribing, and second, provide drug-specific parameters linked to shared care protocols, which could reduce the errors identified and increase patient safety.

\section{Keywords}

clinical decision support system; drug prescribing; general practice; primary health care; specialist drugs.

\section{INTRODUCTION}

In the UK, initiatives such as practicebased commissioning have encouraged the development of GPs with special interests to deliver specialist services in the community. ${ }^{1}$ The introduction of GP commissioning and transfer of the NHS primary care budget to GP consortia ${ }^{2}$ has been seen as an opportunity to change the arrangements for prescribing specialist drugs and providing new services in primary care to avoid patients attending hospital clinics. ${ }^{3}$ Specialist drugs are those that have significant pharmacological complexity and/or rarity of use, making prescribing in primary care relatively uncommon. ${ }^{4}$ In 1991 , the NHS Management Executive outlined core principles and clinical responsibilities associated with prescribing at the primary and secondary care interface, including the use of protocols to support GPs prescribing specialist drugs under shared care agreements. ${ }^{1}$ Local advisory lists were made available to support GPs in managing shifts in prescribing from secondary care to primary care, with medicines grouped as hospital only (red), appropriate for shared care or restricted use (amber), and for GP use and initiation (green). ${ }^{5}$

NHS Prescription Services provides prescribing, financial, and drug information to managing organisations in the NHS in England. In 2006-2007, 1.3 million prescriptions for specialist drugs were

N Chana, DHC, senior primary care pharmacist, NHS Richmond Clinical Commissioning Group, Twickenham, London. T Porat, PhD, research fellow, Department of Primary Care and Public Health Sciences, King's College London, London. C Whittlesea, PhD, professor of pharmacy practice, School of Medicine, Pharmacy and Health, Durham University, Durham. B Delaney, MD, professor of medical informatics and decision making. Department of Surgery and Cancer, Imperial College London, London.

\section{Address for correspondence}

Cate Whittlesea, School of Medicine, Pharmacy issued by GPs, ${ }^{6}$ rising to 1.7 million in 2014-2015, ${ }^{7}$ with the most frequently prescribed specialist drugs (29.3\%) being the immunosuppressant drugs ciclosporin, mycophenolate, sirolimus, and tacrolimus. Between 2005 and 2010 these four drugs were involved in 1103 reported safety incidents, with 282 (25.5\%) directly related to prescribing, and with 11 occurring in primary care (National Patient Safety Agency, personal communication, 2 March 2011). From April 2013, changes in NHS commissioning arrangements for specialist drugs and services have allowed hospitals to repatriate the prescribing of immunosuppressant drugs from primary care to secondary care. ${ }^{8}$

The specialist drugs list maintained by NHS Prescription Services is not exhaustive and does not specify which drugs should be classed as red or amber. This responsibility rests with local organisations such as area prescribing committees, which can lead to regional variation in which specialist drugs are recommended for use only in secondary care or those suitable to be prescribed by GPs under shared care arrangements (in the form of local advisory lists). In 2014$2015,41.89 \%$ of prescriptions for specialist drugs issued by GPs in England were classified as red, that is, hospital only based on the traffic light classification used across northwest London. ${ }^{\text {? }}$

Shared care protocols are developed and Health, Durham University, Queen's Campus, University Boulevard, Stockton-On-Tees, TS17 6BH, UK.

E-mail: cate.whittleseadadurham.ac.uk

Submitted: 28 June 2016; Editor's response: 10 August 2016; final acceptance: 11 October 2016. CBritish Journal of General Practice This is the full-length article (published online 14 Feb 2017) of an abridged version published in print. Cite this version as: Br J Gen Pract 2017; DOI: https://doi.org/10.3399/bjgp17X689389 


\section{How this fits in}

In this study, software engineering and human factor methodologies were used to identify and analyse GPs' current workflow and tasks. Better use of information technology and clinical decision support systems provides a solution to the current risks associated with prescribing specialist drugs, addressing current concerns of GPs and making the process more efficient and less error prone.

and approved by local area prescribing committees such as the Midlands Therapeutic Advisory Committee. ${ }^{10}$ Shared care protocols must be patient specific and clarify individual roles and responsibilities, medicine details, patient monitoring, and circumstances where treatment should be modified or stopped. ${ }^{1}$ One UK survey has reported on the production of 321 shared care protocols that have described 99 different drugs or treatments. ${ }^{11}$ A range of factors influence GP decision making in prescribing specialist drugs, including shared care arrangements and the recommendations of local advisory lists. ${ }^{12} \mathrm{GP}$ dissatisfaction with arrangements for prescribing specialist drugs $^{13}$ and a lack of GP involvement in the development of shared care protocols have been reported. 11,14 Barriers to GP acceptance of shared care protocols have included cost shifting, ${ }^{11,13}$ training, ${ }^{15}$ staffing, and time constraints. ${ }^{16}$ Shared care protocols have been described as variable in standard, lacking formal evaluation, distributed erratically, and with unclear benefits to patients. ${ }^{11}$ Suggested improvements to shared care protocols have included joint agreement between primary care and secondary care ${ }^{15}$ a clearer definition of roles and responsibilities, and dissecting barriers such as risk management and clinical competence. ${ }^{16}$ One potential solution lies in computerised clinical decision support systems (CDSSs).

In the last 30 years, CDSSs have emerged to aid diagnosis, disease management, calculations, and, more recently, supporting electronic prescribing via alerts on drug interactions, allergies, and contraindications. ${ }^{17}$ Systematic reviews of controlled trials of CDSSs in primary care settings have demonstrated positive effects linked to the adherence to clinical guidelines, ${ }^{18,19}$ improved prescribing, ${ }^{20}$ clinician performance, ${ }^{21}$ and drug dosing and therapeutic response.22 However, limitations include a lack of evidence in improving safety measured by medical errors or adverse events, ${ }^{19}$ patient outcomes, ${ }^{23,24}$ and in the understanding of the impact of CDSSs on specific aspects of the prescribing process. ${ }^{25}$ Poor usability has been cited as a core barrier to CDSS adoption, with system designers encouraged to apply common standards based on human-computer interaction methods and user-centred design. ${ }^{26}$

In the UK, commercial CDSSs that support cost-effective prescribing are widely available to $\mathrm{GPs}^{27,28}$ In a recent development CDSSs have become available to support GPs in implementing medicines optimisation. ${ }^{29}$ Such systems are able to provide locally authored messaging platforms to provide drug information and safety alerts to prescribers, including links to local advisory lists known as traffic light classifications and shared care protocols. However, in a recent review, ${ }^{30}$ the impact of one of these systems ${ }^{28}$ has been described as limited compared with other existing cost-effective prescribing initiatives, with drawbacks including alert content and a lack of GP control. No published study has evaluated the potential for a CDSS to support GPs prescribing specialist drugs. In the current study, software engineering and human factors methods were used to identify a potential operating model for a CDSS to support GPs in the safety and quality of specialist drug prescribing.

\section{METHOD}

\section{Study design}

A review of the published literature of CDSSs in the UK identified four stakeholder groups who would provide insight into the requirements of a potential CDSS that could support GPs during the process of prescribing specialist drugs. An exploratory study was initially undertaken using face-to-face interviews with 12 key informants, which were all conducted by the lead trained research pharmacist. The key informants were GPs $(n=2)$, NHS IT managers $(n=2)$, secondary care clinicians $(n=4)$, and representatives from the CDSS industry or GP clinical system suppliers $(n=4)$. Secondary care clinicians with a specific knowledge of specialist drugs and immunosuppression in renal transplantation were invited to participate. UK-based commercial CDSS and GP clinical system suppliers were approached while London-based IT managers and GPs were invited. These interviews were used to gain an understanding of current usage and characteristics of CDSSs available in primary care and to explore the feasibility 
of incorporating a CDSS to support GPs in the prescribing of specialist drugs. Interview schedules were designed for each stakeholder group and used as a topic guide. During each interview emerging themes were used to formulate further questions. Interviews were recorded, transcribed verbatim, and analysed using framework analysis, ${ }^{31}$ supported by NVivo (version 10). The resulting data were reviewed by two members of the research team, and analysed for emerging key themes across the interviews.

In order to further understand the actual needs and requirements of GPs as end users of a potential CDSS, an observational study with GPs was conducted. In total, nine GPs who were users of the three major UK GP clinical systems (EMIS, Vision, and SystmOnel were purposively ${ }^{32}$ selected to participate. The selection took into account the results from the key informant interviews and factors such as GP availability, willingness to participate, and demographic characteristics. Six of the GPs were partners and three were salaried

\section{Box 1. Summary of primary and secondary themes from key informant interviews}

\begin{tabular}{|c|c|c|}
\hline Primary theme & Secondary theme & Key criteria \\
\hline Safety & $\begin{array}{l}\text { Prescribing, monitoring, } \\
\text { specialist drugs, } \\
\text { shared care protocols, } \\
\text { commissioning of } \\
\text { services (secondary } \\
\text { care) }\end{array}$ & $\begin{array}{l}\text { - Communication: problems specifically between } \\
\text { primary care and secondary care (interface) } \\
\text { - Clinical responsibility: for GPs when prescribing } \\
\text { specialist drugs } \\
\text { - Monitoring of patients receiving specialist drugs } \\
\text { - Experiences of specialist drugs (adverse } \\
\text { experiences such as drug interactions, generic } \\
\text { prescribing, repatriation of prescribing to } \\
\text { secondary care) } \\
\text { - Experiences of shared care protocols }\end{array}$ \\
\hline IT systems & $\begin{array}{l}\text { Operational features of } \\
\text { IT systems (primary care) }\end{array}$ & $\begin{array}{l}\text { General usage of computers in general practice } \\
\text { and key characteristics of IT systems } \\
\text { - Regulation (current NHS management systems, } \\
\text { GP clinical system suppliers, and shifting to } \\
\text { hosted systems) } \\
\text { - Data quality (Read coding, accuracy, accreditation) } \\
\text { - Integration of systems } \\
\text { - Implementation of systems } \\
\text { - CDSS and experience in general practice } \\
\text { (current profiles, usage, and characteristics) } \\
\text { - Current and future developments in CDSS } \\
\text { - CDSS and specialist drugs (views and attitudes, } \\
\text { enablers, barriers) }\end{array}$ \\
\hline Cost & $\begin{array}{l}\text { Drug costs, service costs, } \\
\text { IT funding (general } \\
\text { practice), CDSS } \\
\text { funding models }\end{array}$ & $\begin{array}{l}\text { - Prescribing budgets (primary care) } \\
\text { - NHS service costs and use of home care } \\
\text { - GP System of Choice Framework (licence fees), } \\
\text { CDSS funding streams } \\
\text { - CDSS and specialist drugs: funding models }\end{array}$ \\
\hline
\end{tabular}

CDSS = clinical decision support system
GPs. The GPs qualified between 2001 and 2013. All of the GPs described themselves as having either a medium or high IT aptitude. In this observational study the lead researcher discussed and observed the participants perform predefined case studies and took written notes. A research guide was designed to include specific predetermined questions about computer use including CDSSs both during and outside patient consultation, with emerging themes used to formulate further questions. Case scenarios were developed from the results of the key informant interviews with particular emphasis on the use, quality, and availability of shared care protocols. The case scenarios were observed and discussed with each GP, and depicted situations where patients presented prescriptions for specialist drugs (for example, tacrolimus), or these were requested by the hospital, identifying the steps taken by each GP to accept or decline prescribing.

\section{Data analysis}

Data from the observations and discussions were used to develop activity diagrams representing how GPs conducted their work, used their computers both during and outside patient consultation, and how they dealt with the specialist drug prescribing case scenarios. Activity diagrams are often organised as swim lanes to identify who or what is responsible for a specific task or activity, ${ }^{33}$ and have been used to model workflow patterns involved in prescription writing and management in primary care settings ${ }^{34} \mathrm{~A}$ hierarchical task analysis (HTA) was performed to describe in detail the process GPs used to prescribe specialist drugs. HTA models tasks as hierarchies of goals and sub-goals, with plans showing how sub-goals should be undertaken. ${ }^{35}$ Error analysis based on systematic human error reduction and prediction approach (SHERPA) ${ }^{36}$ was applied to the HTA sub-tasks, identifying possible errors, consequence, recovery, probability of occurrence, and remedial action or design solution.

\section{RESULTS}

\section{Exploratory study}

The primary themes that emerged from the exploratory interviews were safety, IT systems, and cost, which are shown in Box 1 with secondary themes and key criteria. In total, 25 CDSSs were identified, of which nine were active systems (provide patientspecific advice), and 16 passive (clinicians need to request the information, not patient- 


\section{Box 2. Key informants' views on the use of specialist drugs and shared care protocols, and the concept of developing a clinical decision support system to support GPs prescribing specialist drugs}

Specialist drugs and shared care protocols

I never had any awareness of shared care protocols, I think they are universally pretty useless and I think

they are a significant area of potential danger.' (GP 1)

I think it's something that we frequently get letters from the hospital consultants ... you know or this is the drug they are taking, it's not made clear to us if we're expected to take on prescribing, it's not made clear to us if there is a shared care protocol in place, it's certainly not clear that they have any idea if there are shared care protocols in place.' (GP 1)

'My normal first response is no, we won't take this on until we get the protocol and when it is sent it is not written in my perspective ... in the sense that it's often written from how it's initiated ... not terribly helpful or easy to use.' (GP 2)

I have written quite a lot of them [shared care protocols] ... usually renal drugs ... once I have written it I send it to our formulary pharmacist ... and then she goes to meetings with the local PCTs ... rather than the individual PCTs now we deal with the sector.' (Secondary care clinician 2)

'I think drug interactions are one of the major headaches that we have with immunosuppressant drugs, you know mainly penicillin-allergic patients come in with a fever and a chest infection and they get clarithromycin and then the next thing you know their tacrolimus levels have doubled.' (Secondary care clinician 3)

'There is a huge variation in bioequivalence, we had one patient who had the wrong brand of tacrolimus and came in and rejected due to lower levels of tacrolimus ... we had another patient who came in who had a recurrence of herpes zoster because she had received a different brand and had been overimmunosuppressed.' (Secondary care clinician 3)

\section{Developing a CDSS to support GPs prescribing specialist drugs}

'I think the priority for me would be to have ... you know, for example, if the shared care protocol is presented to me at the time of prescribing, at the point of prescribing, it's no good having some dim and distant website, somewhere having to trawl through for every drug, every indication, that is useless. '(GP 1)

It has to be intuitive ... it has to be minimally intrusive unless where it needs to be intrusive, so it shouldn't take me 15 minutes for me to do something where previously / would do it in 2 minutes.' (CDSS industry/GP clinical system supplier representative 4]

'The barriers would be there from the big players the clinical applications out there in the practices, EMIS, Vision, SystmOne ... the barriers would be for them for allowing these new guys, this company coming along with this product to talk to them and say yes we can make it work with our product.' (NHS IT manager 1)

So then it can be an extension of the system the GP already has ... which depending on the exact complexity of what you want it is not going to be that expensive ... if you wanted a stand-alone system that was used by the GP for these drugs and by hospital clinicians that initiated the drug it becomes a different cost model altogether, particularly if the hospital records are still paper.' (CDSS industry/GP clinical system supplier representative 2)

And there are substantive costs, not just development but ongoing maintenance of the product and the NHS has not always been able to do that particularly well ... and I think it is very telling ... that the NHS has not chosen to be one of the GP Systems of Choice.' (CDSS industry/GP clinical system supplier representative 4

In order for it be fair to a GP you would have to build in a very high level of supervision in terms of checking ... basically calling the patients back to have their renal function checked and their levels checked after dose changes ... you are basically asking the GP to run a transplant clinic and I think that would be a lot to ask... clearly transplants are about life-sustaining organs ... livers, hearts, and lungs - if you get that wrong then it's good night Vienna.' (Secondary care clinician 4)

CDSS = clinical decision support system. PCT = primary care trust.
Outcomes Framework component of the national General Medical Services contract. In terms of specialist drugs and shared care protocols, mixed experiences were described by the GPs and secondary care clinicians, with specific concerns from a GP perspective in relation to safety, clinical responsibility to prescribe, and in the availability and quality of shared care protocols (Box 2). The concept of developing a CDSS to support GPs prescribe specialist drugs was acknowledged as beneficial by all 12 key informants. Enablers and barriers to CDSS development were identified, including a number of potential operating and funding models (Box 2). Key enablers included multiuser access, active alerting, ease of use, joint development and implementation, patient involvement, data quality and functionality, and the use of existing systems and frameworks. Key barriers included security and regulation, implementation, integration and data quality, and addressing the needs of end users.

\section{Activity diagram and hierarchical task analysis}

GPs described their actions in the case scenarios, deciding whether or not to accept clinical responsibility for prescribing a specialist drug. Figure 1 displays the activity diagram representing their decisionmaking process. The GPs described limited functionality within each GP clinical system to support the prescribing of specialist drugs. All GPs highlighted the importance of adding simple alerts or reminders to the patient's electronic health record (EHR) to indicate that the patient was treated with a specialist drug or that a shared care protocol should be followed. Additional needs included restricting drug quantities and creating tasks, such as recall, to ensure patients were seen on a regular basis. Where shared care protocols were available, all GPs reported they should be scanned and added to the patient's EHR. An additional problem identified was the reliability of administrative staff to scan letters or communications from hospitals. Although the GPs found the use of the clinical systems quick and simple, navigating the systems to locate a shared care protocol was not straightforward (for example, if the scanned letters were not filed by specialty they were difficult to locatel. HTA was applied to the actual task of prescribing specialist drugs and is shown in Box 3. Key steps undertaken were to open the patient's EHR and to familiarise themselves with the patient's clinical history before reviewing the specific). The CDSSs supported a wide range of areas including prescribing, drug information, and disease management. All of the GP clinical systems provided active CDSSs to support prescribing via drug alerts or warnings and reminders or prompts to support the Quality and 


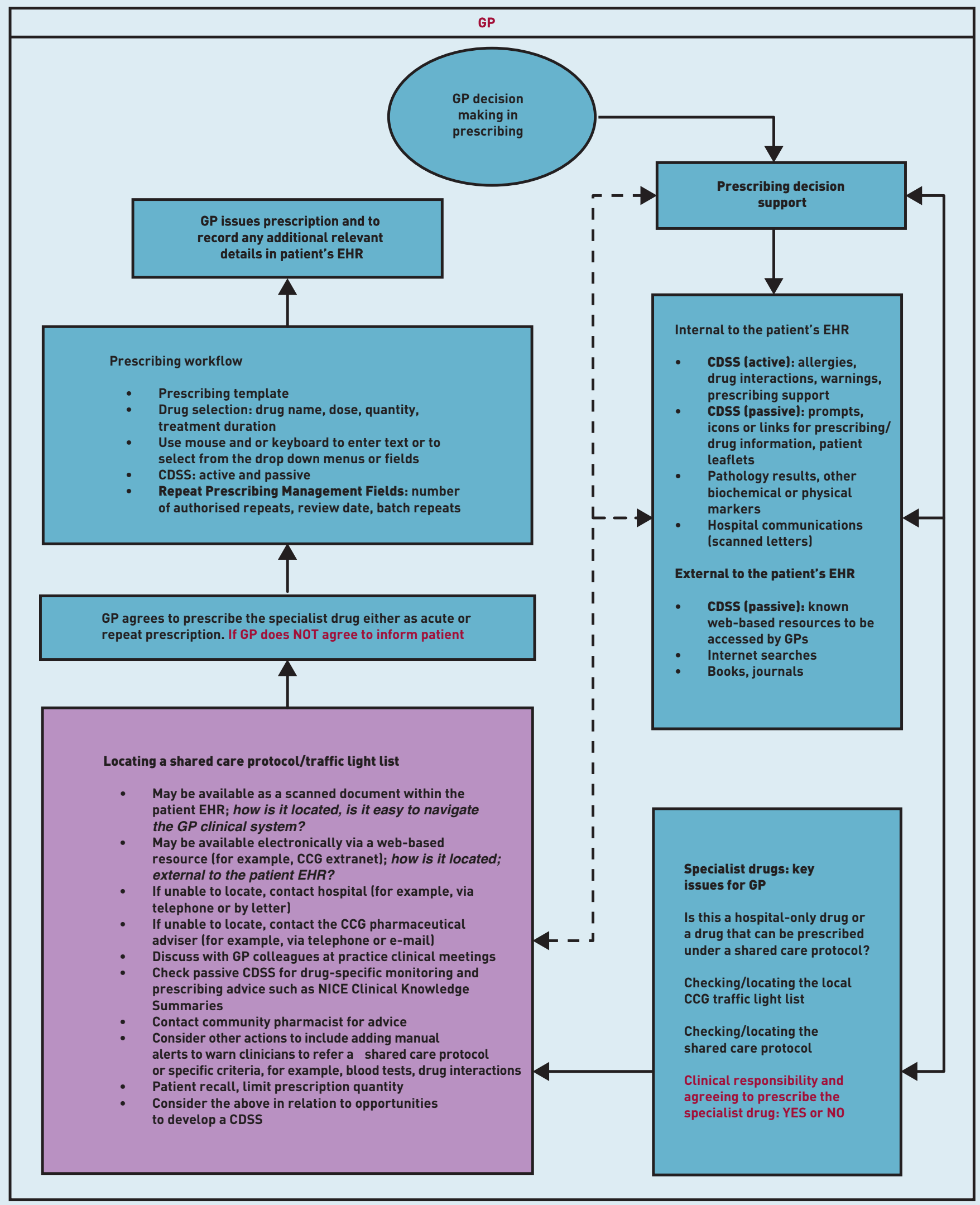

Figure 1. Activity diagram representing GPs' decision making in managing requests to prescribe specialist drugs. $C C G=$ clinical commissioning group. $C D S S=c$ linical decision support system. EHR = electronic health record. 


\section{Box 3. Hierarchical task analysis of the prescribing of specialist drugs by GPs (current tasks)}

\section{Prescribing a specialist drug}

PLAN 0: Either during or outside a patient consultation do 1, 2, and 3 in order.

1. Open patient's electronic health record

2. Get familiar with patient's clinical history

3. Review a request to prescribe a specialist drug

PLAN 3:

Do 3.1 to 3.8 in order according to instructions

3.1. Check traffic light list if drug is 'hospital only'. If 'Yes' go to 3.8. If 'No' go to 3.2

3.2. Check if drug can be prescribed under a shared care protocol. If 'Yes' go to 3.3, if 'No' go to 3.5 or 3.7

3.3. Locate the shared protocol

3.4. Read shared care protocol. If agree to accept clinical responsibility, go to 3.5, if decline, go to 3.7

3.5. Accept clinical responsibility for prescribing the specialist drug

3.6. Prescribe specialist drug

3.7. Decline clinical responsibility for prescribing the specialist drug, go to 3.8

3.8. DO NOT prescribe drug. Refer back to the hospital clinic and inform the patient

request to prescribe a specialist drug. GPs checked if the specialist drug was hospital only' or whether it could be prescribed by using a shared care protocol. These

\section{Box 4. SHERPA error modes}

\begin{tabular}{|c|c|c|}
\hline Error type & Code & Error mode \\
\hline \multirow[t]{10}{*}{ Action errors } & A1 & Operation too long/short \\
\hline & A2 & Operation mistimed \\
\hline & A3 & Operation in wrong direction \\
\hline & $\mathrm{A} 4$ & Operation too little/much \\
\hline & A5 & Misalign \\
\hline & A6 & Right operation on wrong object \\
\hline & A7 & Wrong operation on right object \\
\hline & A8 & Operation omitted \\
\hline & A9 & Operation incomplete \\
\hline & $\mathrm{A} 10$ & Wrong operation on wrong object \\
\hline \multirow[t]{6}{*}{ Checking errors } & $\mathrm{C} 1$ & Check omitted \\
\hline & $\mathrm{C} 2$ & Check incomplete \\
\hline & $\mathrm{C} 3$ & Right check on wrong object \\
\hline & C4 & Wrong check on right object \\
\hline & C5 & Check mistimed \\
\hline & C6 & Wrong check on wrong object \\
\hline \multirow[t]{3}{*}{ Retrieval errors } & $\mathrm{R} 1$ & Information not obtained \\
\hline & R2 & Wrong information obtained \\
\hline & R3 & Information retrieval incomplete \\
\hline \multirow[t]{3}{*}{ Communication errors } & 11 & Information not communicated \\
\hline & 12 & Wrong information communicated \\
\hline & 13 & Information communication incomplete \\
\hline \multirow[t]{2}{*}{ Selection errors } & S1 & Selection omitted \\
\hline & S2 & Wrong selection made \\
\hline
\end{tabular}

SHERPA = systematic human error reduction and prediction approach checks were made by locating and referring to a traffic light classification of specialist drugs. Although CDSS providing support at the point of prescribing was available, for example, ScriptSwitch ${ }^{\circledR}$, none of the GPs referred to this specific functionality during the decision-making process.

\section{Errors involved in prescribing specialist drugs}

The SHERPA analysis demonstrated that although the specific task of prescribing was relatively simple in terms of the number of steps required to complete the task, the potential for error was considerable (Boxes 4 and 5). The potential errors that could occur were either cited by the individual GP (GP1 to GP9) or identified by the research team during data analysis. The underlying problem faced by the GPs was dealing with a paper-based communication system with secondary care. The risk of prescribing specialist drugs in an unsafe manner was increased due to lack of functionality within all of the GP clinical systems and time constraints, particularly where a decision needed to be made during a consultation. To resolve these issues GPs relied on other staff to provide advice and information. In many cases the next opportunity to resolve such problems was when a further prescription was requested by the patient. The predominant theme that emerged from the SHERPA analysis was the high frequency of error mode R1 ('Information not obtained') because information required by the GP was not readily available. The potential adverse outcome was that a GP could inadvertently prescribe a specialist drug classified as hospital only, or prescribe one without reference to an appropriate shared care protocol. In addition, in 11 of the 20 task steps an error rated as a high probability of occurrence could occur. The fundamental problems described by the GPs related to lack of awareness or availability of either the traffic light list or shared care protocols. In addition, where shared care protocols were required, a major obstacle was locating them in either a paper or electronic format, particularly during patient consultations.

It was evident throughout the whole process that GP clinical systems and CDSSs were not able to provide full solutions to the problems described by the GPs. One remedial solution was to manually add simple alerts or reminders to the patient's EHR. These alerts allowed free text to be added to a pop-up box informing each user of key messages related to prescribing of specialist drugs, for example, checking 


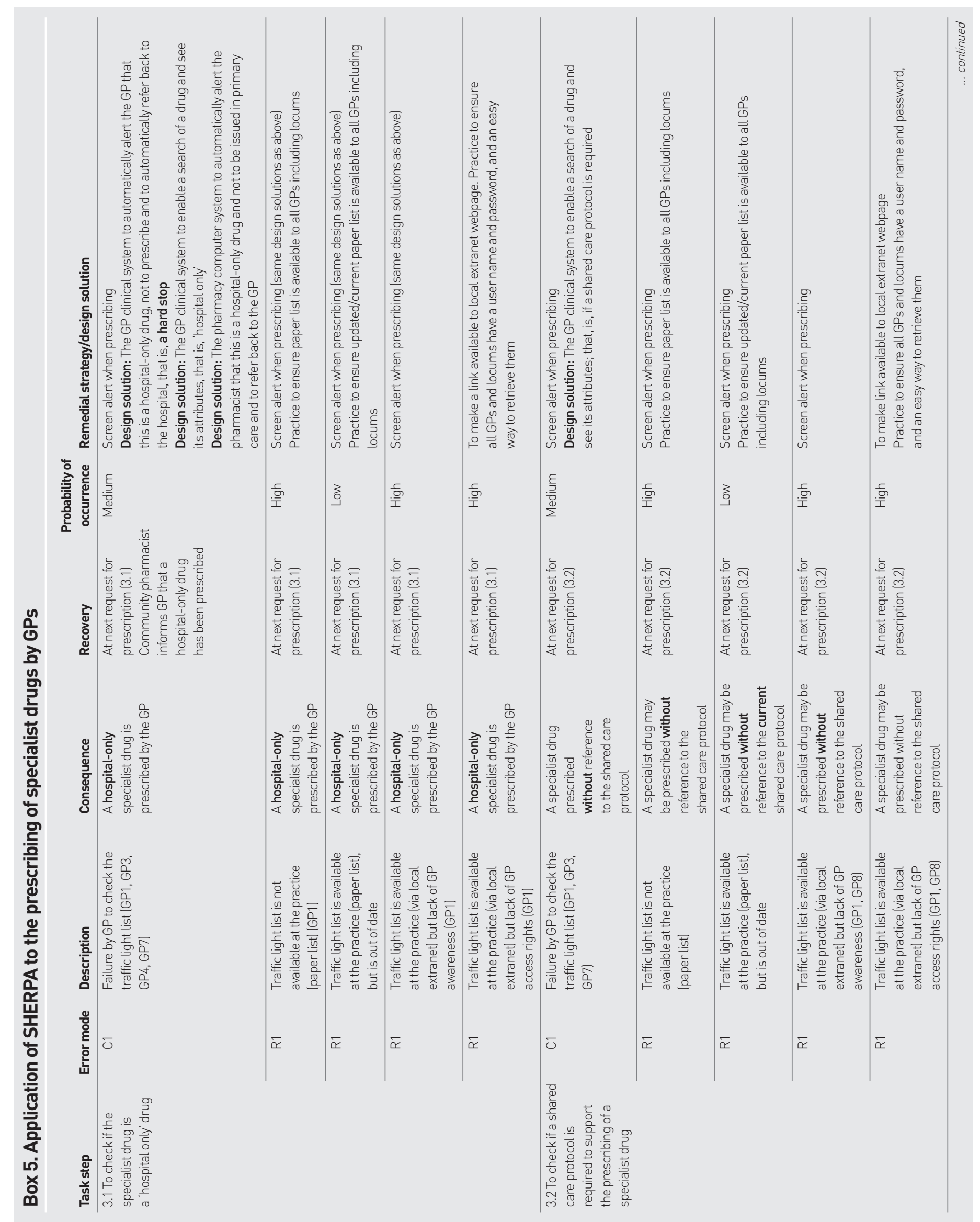




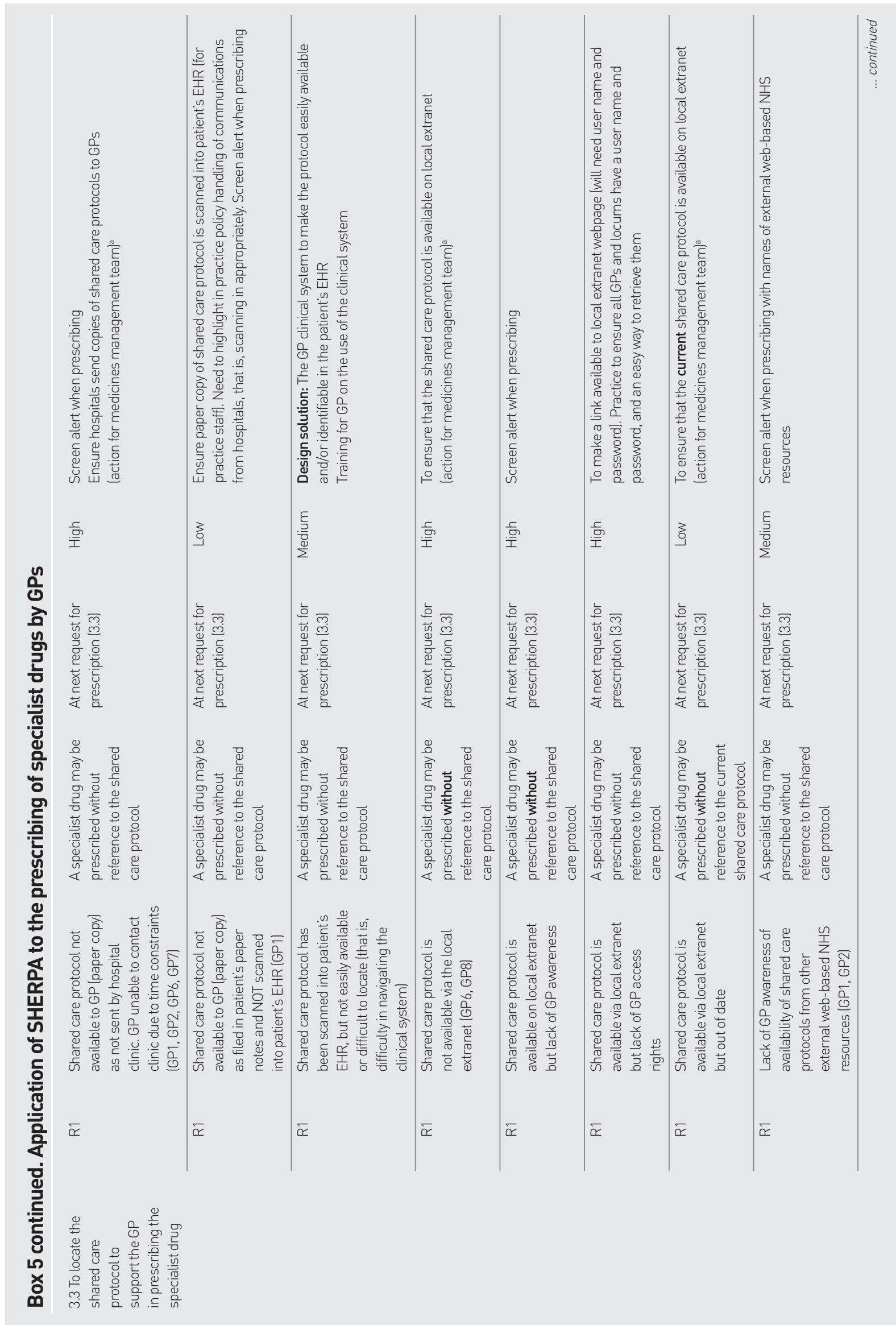




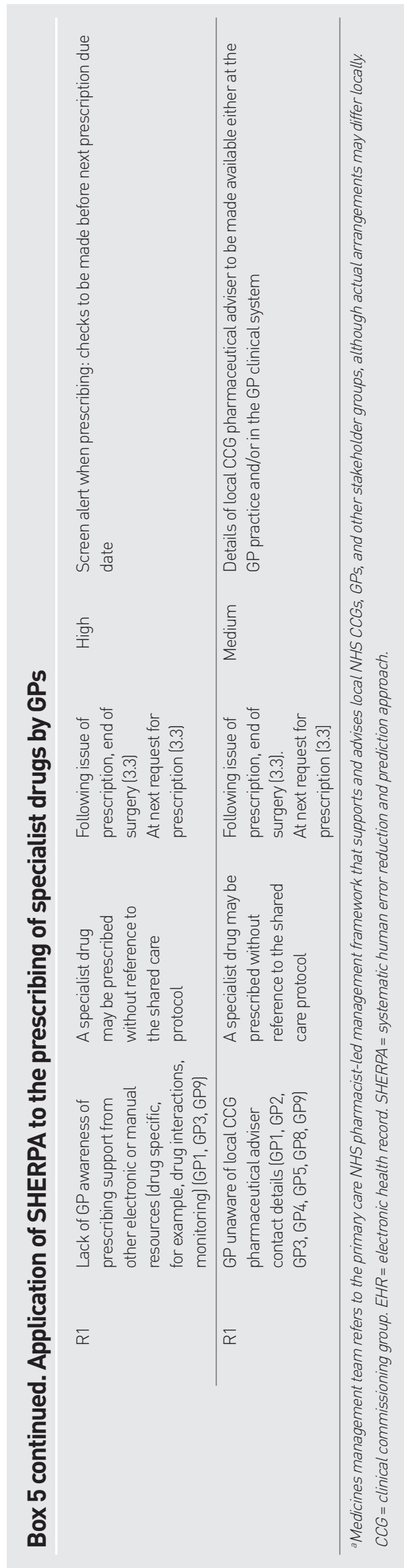

the shared care protocol or requirements for regular blood tests. Other interventions described were manual tasks to contact and liaise with a range of individuals, such as other GPs, primary care pharmacists, and clinical teams in secondary care. Application of both HTA and SHERPA identified a potential operating model for a CDSS to support GPs in prescribing specialist drugs. Box 6 describes an HTA of this proposed model, which demonstrates how GPs could potentially safely prescribe a specialist drug in primary care.

\section{DISCUSSION}

\section{Summary}

Previous studies have identified concerns from GPs in prescribing specialist drugs and in the use of shared care protocols, ${ }^{11-13}$ Difficulties have included lack of awareness or access to either local traffic light classification lists or shared care protocols. ${ }^{11}$ Furthermore, CDSSs have been reported to appear too late during patient consultations, by which time GPs had already made a decision around treatment options. ${ }^{37}$ In the current study it was identified that the lack of specific functionality in GP clinical systems (EMIS, Vision, SystmOne), including CDSSs, adversely affected the GPs' ability to resolve problems associated with prescribing of specialist drugs. To the authors' knowledge, this is the first study to perform a detailed analysis of the tasks and potential errors (HTA and SHERPA) during the prescribing process in the three major GP clinical systems available in the UK. This detailed analysis enabled the identification of specific weaknesses and potential errors with current systems that have not been previously identified, and the proposal of possible solutions in the form of a potential operating model for a CDSS to support GPs in prescribing specialist drugs.

\section{Strengths and limitations}

The strength of this work resides in its use of human factors methods (HTA and SHERPAl for analysing the GPs' tasks and workflows in prescribing specialist drugs. Using human factors techniques and risk assessment methods are encouraged for designing healthcare interventions. ${ }^{38,39}$ Nevertheless, this is the first study that has analysed the prescribing of specialist drugs by GPs using these methods. Participants used the three major GP clinical systems available in the UK. This ensures that the findings are relevant to most GP practices. However, the recruitment process of the participants was not optimal as two of the GPs (GP1, GP2) participated in both 


\section{Box 6. Hierarchical task analysis for prescribing specialist drugs by GPs (potential operating model supported by CDSS)}

\section{Prescribing a specialist drug}

PLAN 0: Either during or outside a patient consultation do 1, 2, and 3 in order.

1. Open patient's electronic health record

2. Get familiar with patient's clinical history

3. Review a request to prescribe a specialist drug PLAN 3:

Do 3.1 to 3.10 in order and according to instructions

3.1. Enter the 'Management of Specialist Drugs Module' integrated within the electronic health record

3.2. Enter drug name and check drug attributes

3.3. If drug is 'hospital only' go to 3.10

3.4. If drug requires a shared care protocol go to 3.5

3.5. Module will automatically update fields for monitoring requirements according to the information in the patient's electronic health record

3.6. If data are not available in patient's electronic health record, module will automatically prompt action or task, for example, full blood count

3.7. Decide whether to accept clinical responsibility for prescribing the specialist drug. If agree to accept clinical responsibility go to 3.8 , if decline, go to 3.9

3.8. Follow the prescribing workflow and issue prescription (consider repeat or acute prescription)

3.9. Decline clinical responsibility for prescribing the specialist drug, go to 3.10

3.10. Exit module, system will automatically refer to hospital clinic, inform the patient

3.11. Module will save all recorded information with relevant Read codes in the health record on exit and automatically update the prescribing authorisation and/or review date

3.12. If GP does not enter the 'Management of Specialist Drugs Module' and prescribes a 'hospital only' drug or prescribes a drug without reference to the shared care protocol, the GP clinical system will automatically alert the GP

CDSS = clinical decision support system

\section{Funding}

This study was conducted as part of the Doctorate in Healthcare (Clinical Practice and Medication Use) that was awarded to Dr Narinder Singh Chana by King's College London in November 2015. Contributions towards course fees were received from Takeda Pharmaceuticals and GSK Pharmaceuticals.

\section{Ethical approval}

Ethical approval for the key informant interviews (Reference BDM/11/12-82) was granted by King's College London Biomedical Sciences, Dentistry, Medicine and Natural \& Mathematical Sciences Research Ethics Subcommittee on 28 May 2012. A modification of this application to include the observational study and interviews with GPs was granted on 9 May 2014

\section{Provenance}

Freely submitted; externally peer reviewed.

\section{Competing interests}

The authors have declared no competing interests.

\section{Discuss this article}

Contribute and read comments about this article: bjgp.org/letters the early stages of technology development, before more detailed processes of interface design are undertaken. Furthermore, although HTA was chosen as the preferred research method in this study, the results from the SHERPA analysis identified that further exploration of the decision-making processes used by GPs is required. This could be undertaken by adopting a cognitive approach to task analysis to inform further research in this area. It is also common that repeat prescribing and tasks relating to hospital correspondence are undertaken separately from the patient consultation.

\section{Comparison with existing literature}

Although health care has increased the awareness of retrospective safety assessment techniques such as root cause analysis, the use of predictive safety assessment techniques (such as SHERPA or failure modes and effects analysis) is limited and there is a need to adopt more proactive and rigorous approaches..$^{40,41}$ This work is novel as no previous study has applied HTA and SHERPA to inform a CDSS for prescribing specialist drugs. Previously reported use of HTA with or without SHERPA in the UK has been primarily limited to the study of endoscopic surgery, ${ }^{42}$ diagnosis, ${ }^{43}$ drug administration, ${ }^{44}$ and anaesthesia use. ${ }^{45}$

\section{Implications for research and practice}

The results from this study have highlighted that, despite the availability of CDSSs and other functionality in GP clinical systems, safety concerns remain with the use of specialist drugs prescribed by GPs in primary care. Designing a CDSS that considers the needs of GPs, their current workflow, and their decisionmaking process has the potential to both facilitate the prescribing of specialist drugs and decrease the associated risks with prescribing errors. Designing and evaluating with GPs a CDSS prototype, based on the authors operating model, could be a meaningful step in improving the current prescribing process. In addition, further research could investigate how GP clinical systems and community pharmacy systems could incorporate active hard stops to alert users when hospital-only drugs are prescribed. 


\section{REFERENCES}

1. Khambh J, Barnick C. Shared-care arrangements and the primary/secondarycare interface. Prescriber 2007; 18(7): 23-29.

2. Department of Health. Equity and excellence: liberating the NHS. 2010. https:// www.gov.uk/government/uploads/system/uploads/attachment_data/file/213823/ dh_117794.pdf (accessed 13 Jan 2017).

3. [No authors listed]. Moving specialised medicines out of hospital. Drug Ther Bull 2012; 50(11): 121.

4. Coventry and Warwickshire Area Prescribing Committee. Specialist drugs list. http://www.coventrywarksapc.nhs.uk/Specialist-Drugs-List/Specialist-DrugsList laccessed 23 Jan 2017).

5. Stephens M. Strategic medicines management. London: Pharmaceutical Press, 2005

6. NHS Business Services Authority. NHS Prescription Services. ePACT.net. 2012. [Guest user]. http://uww.nhsbsa.nhs.uk/PrescriptionServices/3230.aspx laccessed 23 Jan 2017).

7. NHS Business Services Authority. Information services portal. [Guest user]. 2016. https://apps.nhsbsa.nhs.uk/infosystems/welcome laccessed 23 Nov 2016).

8. Devaney A, Lee M. The use of generic immunosuppression for transplant patients: a UK perspective. Eur J Hosp Pharm 2013; 20: 272-274.

9. North West London Integrated Formulary New Drugs Panel. North West London Red List - medicines that hospital doctors should not ask GPS to prescribe. Version 27. June 2014. http://www.hounslowccg.nhs.uk/media/38282/ nwl-red-list-june14-vs-27.pdf (accessed 13 Jan 2017).

10. Midlands Therapeutics Review and Advisory Committee. Independent review of medicines for primary care. http://ccg.centreformedicinesoptimisation.co.uk/ mtrac/ laccessed 13 Jan 2017).

11. Duggan C, Beavon N, Bates I, Patel S. Shared care in the UK: failings of the past and lessons for the future. Int J Pharm Pract 2001; 9(3): 211-216.

12. Crowe S, Tully MP, Cantrill JA. The prescribing of specialist medicines: what factors influence GPs' decision making? Fam Pract 2009; 26(4): 301-308.

13. Horne R, Mailey E, Frost S, Lea R. Shared care: a qualitative study of GPs' and hospital doctors' views on prescribing specialist medicines. Br J Gen Pract 2001; 51(464): 187-193.

14. Gerada C, Tighe J. A review of shared care protocols for the treatment of problem drug use in England, Scotland, and Wales. Br J Gen Pract 1999; 49(439): 125-126.

15. Salt N, Parkes E, Scammell A. GPs' perceptions of the management of ADHD in primary care: a study of Wandsworth GPs. Prim Health Care Res Dev 2005; 6: 162-171.

16. Iliffe S, Wilcock J, Haworth D. Obstacles to shared care for patients with dementia: a qualitative study. Fam Pract 2006; 23(3): 353-362.

17. Barber N. Designing information technology to support prescribing decision making. Qual Saf Health Care 2004; 13(6): 450-454

18. Eslami S, Abu-Hanna A, de Keizer NF. Evaluation of outpatient computerized physician medication order entry systems: a systematic review. J Am Med Inform Assoc 2007; 14(4): 400-406.

19. Jamal A, McKenzie K, Clark M. The impact of health information technology on the quality of medical and health care: a systematic review. HIM J 2009; 38(3): 26-37.

20. Schedlbauer A, Prasad V, Mulvaney C, et al. What evidence supports the use of computerized alerts and prompts to improve clinicians' prescribing behavior? J Am Med Inform Assoc 2009; 16(4): 531-538.

21. Garg AX, Adhikari NK, McDonald $\mathrm{H}$, et al. Effects of computerized clinical decision support systems on practitioner performance and patient outcomes: a systematic review. JAMA 2005; 293(10): 1223-1238.

22. Durieux $P$, Trinquart $L$, Colombet I, et al. Computerized advice on drug dosage to improve prescribing practice. Cochrane Database Syst Rev 2008; (3):
CD002894.

23. Sintchenko V, Magabri F, Tipper S. Are we measuring the right end-points? Variables that affect the impact of computerised decision support on patient outcomes: a systematic review. Med Inform Internet Med 2007; 32(3): 225-240.

24. Mollon B, Chong JJR, Holbrook AM, et al. Features predicting the success of computerized decision support for prescribing: a systematic review of randomized controlled trials. BMC Med Inform Decis Mak 2009; 9: 11.

25. Pearson SA, Moxey A, Robertson J, et al. Do computerised clinical decision support systems for prescribing change practice? A systematic review of the literature (1990-2007). BMC Health Serv Res 2009; 9: 154

26. Horsky J, Schiff GD, Johnston D, et al. Interface design principles for usable decision support: a targeted review of best practices for clinical prescribing interventions. J Biomed Inform 2012; 45(6): 1202-1216.

27. [No authors listed]. The computer says 'no'? Drug Ther Bull 2010; 48(4): 37.

28. Optum. ScriptSwitch. 2016. http://www.scriptswitch.com/ laccessed 13 Jan 2017).

29. First Databank. OptimiseRx solution overview. 2014. http://www.valeofyorkccg nhs.uk/rss/data/uploads/prescribing/optimise/overview.pdf laccessed 13 Jan 2017).

30. Hire S, Rushforth B. General practitioners' views on using a prescribing substitution application (ScriptSwitch ${ }^{\circledR}$ ). Inform Prim Care 2013; 21(1): 1-11.

31. Ritchie J, Spencer L. Qualitative data analysis for applied policy research. In: Bryman A, Burgess RG, eds. Analyzing qualitative data. Abingdon: Routledge, 1994: 173-194.

32. Marshall MN. Sampling for qualitative research. Fam Pract 1996; 13(6): $522-$ 525

33. Benson T. Principles of health interoperability HL7 and SNOMED. 2nd edn London: Springer, 2012

34. Johnson KB, Fitzhenry F. Case report: activity diagrams for integrating electronic prescribing tools into clinical workflow. J Am Med Inform Assoc 2006; 13(4): 391-395.

35. Shepherd A. Hierarchical task analysis. London: Taylor \& Francis, 2001.

36. Embrey DE. SHERPA: a systematic human error reduction and prediction approach. In: Proceedings of the international topical meeting on advances in human factors in nuclear power systems. Knoxville, TN: American Nuclear Society, 1986.

37. Hayward J, Thomson F, Milne H, et al. 'Too much, too late': mixed methods multi-channel video recording study of computerized decision support systems and GP prescribing. J Am Med Inform Assoc 2013; 20(e1): e76-e84.

38. National Patient Safety Association: National Reporting and Learning Service. Lessons from high hazard industries for healthcare - full report. 2010. http:// wuw.nrls.npsa.nhs.uk/resources/?entryid45=74930\&p=7 laccessed 13 Jan 2017).

39. NHS. National Patient Safety Association. Risk assessment programme: overview. 2006. http://uww.nrls.npsa.nhs.uk/resources/?entryid45=59813\&p=15 laccessed 13 Jan 2017).

40. Sujan M, Spurgeon P, Cooke M, et al. The development of safety cases for healthcare services: practical experiences, opportunities and challenges. Reliability Engineering and System Safety 2015; 140: 200-207.

41. Pippias M, Tomson CR. Patient safety in chronic kidney disease: time for nephrologists to take action. Nephrol Dial Transplant 2014; 29(3): 473-475.

42. Joice $P$, Hanna GB, Cuschieri A. Errors enacted during endoscopic surgery: a human reliability analysis. Appl Ergon 1998; 29(6): 409-414.

43. Porat T, Kostopoulou O, Woolley A, Delaney BC. Eliciting user decision requirements for designing computerized diagnostic support for family physicians. J Cogn Eng Decis Mak 2015; 10: 57-73.

44. Lane R, Stanton NA, Harrison D. Applying hierarchical task analysis to medication administration errors. Appl Ergon 2006; 37(5): 669-679.

45. Phipps D, Meakin GH, Beatty PC, et al. Human factors in anaesthetic practice: insights from a task analysis. Br J Anaesth 2008; 100(3): 333-343. 